\title{
Le capital social des filières : une dimension essentielle de leurs capacités d'adaptation. Illustration avec la filière comté
}

\author{
Cyrille Rigolot ${ }^{*}$
}

UMR 1273 Métafort AgroParisTech-Inra-Irstea-VetAgroSup, Inra, route de Theix, 63122 Saint-Genès-Champanelle, France

\begin{abstract}
Résumé - Dans le contexte actuel très évolutif et marqué par une crise profonde du secteur laitier, la filière comté est décrite comme un exemple de réussite économique. En prenant cette filière comme illustration, cet article propose d'explorer l'importance du capital social dans les capacités d'adaptation des filières. Après avoir défini ces termes, nous présentons le capital social de la filière comté, qui combine différents types de liens sociaux entre ses acteurs, avec son unité coopérative de base (la fruitière) et son interprofession spécifiques. En nous basant sur le journal interne de l'interprofession et en complément de la littérature, nous montrons comment ces liens sociaux permettent d'adapter les volumes produits à la demande du marché, de façon à maintenir des prix du lait élevé. Ensuite, nous montrons que ce capital social peut être mobilisé comme source d'innovation pour d'autres problématiques, en prenant des exemples concrets dans le domaine social (évolution du travail des fromagers) et technique (améliorations de la qualité du fromage et autonomie des exploitations). Le capital social de la filière présente des risques d'érosion, mais il permet aussi de lutter, en quelque sorte, contre sa propre dégradation. Ces éléments dynamiques sont discutés en conclusion, ainsi que les perspectives de cette étude et des enseignements plus généraux.
\end{abstract}

Mots clés : comté / fromage à pâte dure / filière / capital social / capacité d'adaptation

\begin{abstract}
The social capital of value chains: a key dimension of their adaptive capacities. Illustration with the "comté" cheese value chain. In the current period of milk crisis, the "comté" cheese value-chain is described as an example of economic success. Considering this value-chain as an illustration, this paper explores the importance of social capital to enhance adaptive capacities. After defining these concepts, we present the social capital of the "comté" value-chain, combining different types of social links between its actors, with its specific small cooperative units ("fruitières") and inter-professional organization. On the basis of the internal journal of the value-chain and the literature, we show how these social links enable to adapt cheese production to the demand, in order to maintain high milk price. Furthermore, we show that social capital can also be used as a source of innovation for other issues, with practical examples in the social domain (evolutions of cheese markers' working conditions) and in the technical domain (improvement of cheese quality and farm self-sufficiency). The social capital of the value chain is exposed to a risk of erosion, but at the same time it enables to cope with this risk of its own erosion. These dynamic perspectives are discussed in the conclusion, as well as perspectives and general lessons.
\end{abstract}

Keywords: comté / hard cheese / value chain / social capital / adaptive capacity

\section{Introduction}

Les années 2015 et 2016 ont été marquées par une profonde crise de l'élevage laitier français. La diminution du prix du lait a mis en difficulté un nombre considérable d'éleveurs, en prise par ailleurs avec des investissements lourds et des charges élevées. Cette crise coïncide avec la fin des quotas laitiers, qui pourrait

\footnotetext{
* Auteur de correspondance :

cyrille.rigolot@clermont.inra.fr
}

renforcer encore à l'avenir les mécanismes de baisse tendancielle du prix du lait standard (Dervillé et Allaire, 2014). Dans ce contexte de crise généralisée, certaines filières laitières semblent toutefois mieux résister que d'autres. C'est le cas de la filière d'appellation d'origine contrôlée (AOC) du comté, qui arrive à maintenir des prix du lait de l'ordre de 30 à $40 \%$ plus élevés que le prix standard national tout en maîtrisant relativement bien ses charges (Ricard, 2009). Cette réussite économique de l'AOC comté n'est pas seulement conjoncturelle, mais semble confirmer une tendance depuis les années 1990-2000 (Kroll, 2008). Sur les plans environnementaux et sociaux, la filière 
comté est également aujourd'hui souvent mise en avant, pour avoir réussi plus qu'ailleurs à maintenir des systèmes relativement extensifs et un tissu rural dense et dynamique, avec un taux de renouvellement de la population d'éleveurs parmi les plus élevés de France (Fumey et Bérion, 2010 ; Elisseef, 2015).

Plusieurs auteurs ont montré que la réussite économique de l'AOC comté tient à la création d'une rente d'appellation et au partage équitable de cette rente (Jeanneaux et al., 2011). La rente d'appellation est liée à un cahier des charges rigoureux, garant d'une qualité spécifique du produit. Des éléments structurants sont notamment l'alimentation au foin, l'utilisation de la race montbéliarde, le travail au lait cru et l'affinage long (Ricard, 1994). Cependant, l'existence d'une qualité spécifique n'est pas suffisante à elle seule pour expliquer la rente d'appellation. Il faut encore que cette qualité soit reconnue (réputation) et qu'elle se traduise dans les faits par des prix rémunérateurs. Pour maintenir des prix du lait élevés, la maîtrise des volumes produits en cohérence avec la demande du marché est essentielle. Jeanneaux et al. (2009) expliquent cette maîtrise de l'offre par une diversité d'outils économiques et contractuels, tandis que Torre (2002) fait appel à la notion de « confiance organisationnelle ».

En plus de cette rente d'appellation, les capacités d'évolution et d'adaptation de la filière dans un contexte très évolutif sont aussi très importantes. En effet, le secteur laitier doit faire face à des évolutions et des incertitudes majeures sur les plans techniques, économiques, climatiques... (Dedieu et Ingrand, 2010). Selon cette perspective, la réussite de la filière comté est souvent associée à sa faculté à concilier "tradition » et « modernité », autrement dit stabilité et changement. Dans le présent article, nous soutenons qu'une dimension essentielle de cette capacité d'adaptation particulière est le capital social de la filière. Le concept de « capital social » considère que les relations sociales sont une ressource mobilisable (Adler et Kwon, 2002). Initialement proposé en sociologie, ce concept a été repris en économie et en géographie, avec la notion de capital social territorial (Angeon et Callois, 2005). En revanche, le capital social n'a pas encore été utilisé à notre connaissance pour décrire des filières agroalimentaires, a fortiori laitières. Le capital social d'une filière tel que nous l'entendons et le capital social territorial présentent un certain recouvrement, plus ou moins grand selon le degré d' « ancrage territorial » de la filière (Rieutort, 2006). Cependant, alors que le capital social territorial est lié à des indicateurs comme la vie associative, civique et communautaire dans le territoire (Angeon et Callois, 2005), le capital social d'une filière est centré autour d'un produit.

Après avoir défini le capital social et ses liens avec les capacités d'adaptation en général, nous montrons que la filière comté possède un capital social bien particulier, articulant étroitement différents types de liens sociaux. Ensuite, pour la problématique de la maîtrise des volumes produits essentielle au maintien de la rente d'appellation, nous détaillons comment ce capital social est mobilisé pour s'adapter à la demande fluctuante du marché, en reformulant et complétant les analyses antérieures (Torre, 2002 ; Jeanneaux et al., 2009). Nous montrons ensuite l'intérêt adaptatif du capital social dans des domaines social et technique. Pour ceci, nous nous appuyons sur des exemples concrets d'adaptation reportés depuis 1993 dans «Les Nouvelles du Comté », le journal interne de l'interprofession sur la vie de la filière (quatre numéros par an,
92 numéros au total, CIGC, 2016). Les Nouvelles du Comté sont référencées dans cet article par la notation $\mathrm{NdC}$ suivie du numéro, permettant au lecteur d'accéder à tout le détail technique nécessaire. Le choix de cette source est lié au caractère exploratoire de notre étude (montrer qualitativement l'importance du capital social pour une filière) et la volonté d'accéder à un point de vue interne porté par les acteurs sur euxmêmes. En conclusion, nous discutons les dynamiques du capital social de la filière et des enseignements plus généraux.

\section{Capital social et capacités d'adaptation}

\subsection{Définition et caractérisation du capital social}

Parmi les nombreuses définitions existantes (Angeon et Callois, 2005), nous retiendrons dans le présent article que le capital social est « l'ensemble des normes et des réseaux qui facilitent l'action collective » (Woolcok et Narayan, 2000). Selon cette définition, le capital social est donc composé à la fois par des «normes », qui renvoient à un ensemble de règles informelles et aux valeurs des individus, et par des « réseaux », qui renvoient à ses caractéristiques structurelles (Putnam, 1995 ; Angeon et Callois, 2005). Cette définition est compatible avec celle de Lin (2001), pour qui le capital social est « une richesse potentielle incorporée dans la structure sociale et qui peut être (mais ne l'est pas nécessairement) mobilisée en cas de besoin ». Dans les recherches sur le développement territorial, le capital social est abordé en considérant différents types de liens, pour distinguer entre « le capital social qui unit (bonding), qui lie (linking) et qui relie (bridging) » (Woolcok et Narayan, 2000). Les liens de type bonding unissent des individus au sein d'une même communauté et sont qualifiés d'horizontaux. Les liens de type linking, de nature verticale, caractérisent des interactions entre des agents appartenant à des groupes différents. Enfin, dans les relations de type bridging, les agents sont distants les uns des autres (Angeon et al., 2006). En faisant un rapprochement avec le concept de proximité, Angeon (2008) a montré que les liens bonding reposaient plutôt sur une logique de similitude, les liens linking sur une logique d'appartenance, et les liens bridging sur une logique de relation à distance, reposant sur une communauté d'intérêt entre les acteurs.

\subsection{Avantages et inconvénients du capital social pour les capacités d'adaptation}

Le capital social favorise la collecte et la circulation de l'information, qui sont des éléments déterminants pour faciliter l'innovation collective (Angeon et al., 2006 ; Alguezaui et Filieri, 2010). Les innovations collectives permettent de renforcer les capacités d'adaptation des systèmes de production aux aléas, en particulier climatiques (Adger, 2003). Le capital social peut aussi fournir à celui qui le possède de l'influence, du pouvoir et des possibilités de contrôle (Adler et Kwon, 2002). Il est également lié à la solidarité entre acteurs, et à ce que Chia (2008), dans le domaine de l'élevage, a qualifié de «flexibilité relationnelle ». Une notion importante est celle d' « appropriabilité » (Adler et Kwon, 2002), selon laquelle des relations sociales a priori liées à un objectif donné peuvent être potentiellement mobilisées pour d'autres objectifs. Cependant, le capital social a aussi des inconvénients (coûts d'entretien élevés, obligations morales contraignantes, risque de repli sur 


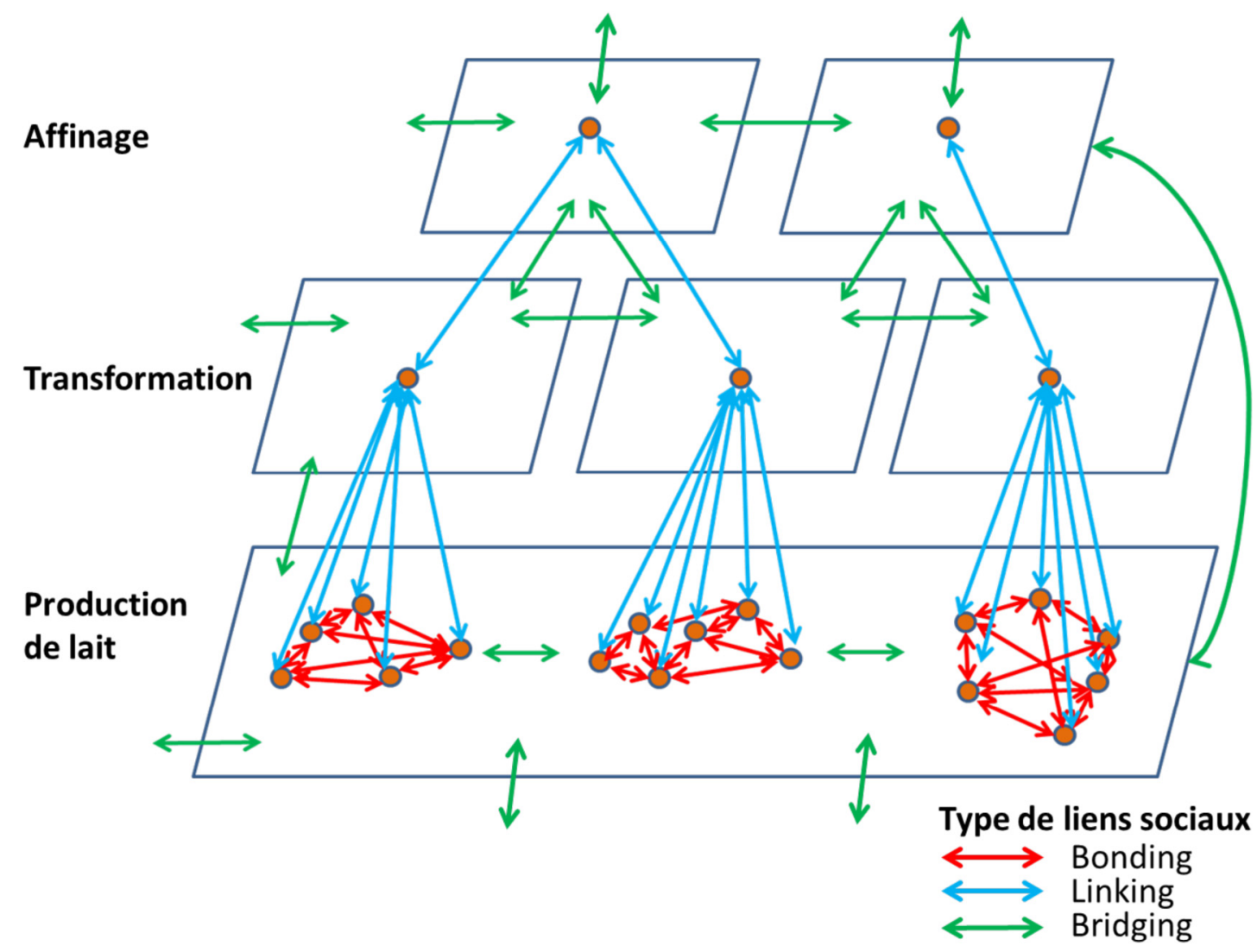

Fig. 1. Architecture de base du capital social de la filière comté, combinant différents types de liens sociaux. Cette organisation doit être vue de façon dynamique, articulée avec une multitude d'acteurs et une forte hétérogénéité non représentées sur le schéma.

Fig. 1. Basic architecture of the social capital of the "comté" cheese value-chain, combining different types of social links. This organization must be seen as a dynamic entity, articulated with numerous actors and a high level of heterogeneity not represented in the figure.

soi-même des acteurs...). En fait, la balance entre les bénéfices et les risques du capital social vis-à-vis des différents types d'innovation dépend notamment des caractéristiques structurelles du réseau (importance relative des liens de type bonding, bridging et linking). En particulier, une forte cohésion permet de développer la confiance entre acteurs, de réduire les comportements opportunistes et de mieux partager les risques. En revanche, dans certaines conditions, elle peut limiter les capacités d'initiative des individus et créer des situations de verrouillage (Alguezaui et Filieri, 2010). Dans le domaine des recherches sur le développement territorial, Angeon et Callois (2005) montrent qu'un excès de localisme peut dans une certaine mesure être néfaste au développement. Ces auteurs insistent sur la «force des liens faibles », c'est-à-dire l'importance d'avoir des relations ouvertes sur l'extérieur, et la nécessité de considérer les combinaisons entre différents types de liens sociaux (Angeon et Callois, 2005).

\section{Capital social et capacité d'innovation de la filière comté}

\subsection{Particularités du capital social de la filière comté}

La filière comté est organisée en trois grandes étapes avec des groupes d'acteurs distincts (Vernus, 1988 ; Ricard, 1994 ; Fumey et Bérion, 2010 ; Jeanneaux et al., 2011) (Fig. 1) :
- la production de lait par les agriculteurs (environ 2600 exploitations);

- la transformation du lait en " fromage en blanc », c'est-àdire pas encore ou peu affiné. Les fromagers sont les acteurs emblématiques de cette étape ;

- l'affinage du fromage, avec une diversité de métiers spécifiques (Torre, 2002).

La production de comté reste majoritairement contrôlée par les producteurs de lait par le biais des coopératives, qui assurent encore environ les trois quarts de la production (Kroll, 2008). Ces coopératives coexistent avec des établissements de statut privé (artisans fromagers et grands groupes extérieurs à la région). La gouvernance de la filière est assurée par le Comité interprofessionnel du gruyère de comté (CIGC), composé de représentants des trois collèges producteurs, transformateurs et affineurs, et qui joue le rôle d'organisme de défense et de gestion. Le CIGC a dans ses objectifs la publicité, la recherche technique, la défense de l'appellation d'origine, l'administration et la gestion du marché $\left(\mathrm{NdC} \mathrm{n}^{\circ} 21\right)$.

À la base, les agriculteurs d'une même fruitière partagent des liens particulièrement forts (de type bonding). De façon très significative, ils se définissent non pas seulement comme producteurs de lait, mais d'abord comme " producteurs de fromage » $\left(\mathrm{NdC} \mathrm{n}^{\circ} 63\right)$. Cette implication dans la chaîne de valeur crée aussi un lien très fort (linking) entre les 
coopératives et les affineurs. Cette relation est d'abord basée sur l'échange et la volonté de valoriser collectivement le produit, et non pas sur un rapport de subordination ou de domination (comme cela a été observé dans d'autres filières laitières (Ricard, 1994 ; Jeanneaux et al., 2011). Enfin, l'ensemble des opérateurs entretiennent des relations à distance sur la base de leur intérêt commun, même s'ils n'ont pas nécessairement des échanges réguliers. Ces liens de bridging peuvent être soit internes à la filière, soit entre la filière et son environnement au sens large (consommateurs, autres secteurs régionaux, institutions, autres filières fromagères...) (Fig. 1). Les liens sociaux des trois types peuvent prendre différentes formes : règles plus ou moins formelles, interactions diverses, journal interne $(\mathrm{NdC})$, évènements festifs ( $\mathrm{NdC} \mathrm{n}^{\mathrm{o}}$ 62).

\subsection{Mobilisation du capital social pour s'adapter à la demande du marché (maîtrise de l'offre)}

La maîtrise des volumes produits a été une préoccupation importante dans l'histoire de l'AOC (et d'actualité avec la fin des quotas laitiers depuis 2015, $\mathrm{NdC} \mathrm{n}^{\mathrm{o}} 87$ ). Nous allons montrer que cette maîtrise mobilise les trois types de liens : bonding, linking et bridging (Tab. 1). Selon l'analyse de Jeanneaux et Perrier-Cornet (2011), un élément essentiel est l'entente du tandem fruitière-affineurs (type linking), considérée comme structurante. Cette entente se matérialise par un contrat type, permettant le pilotage institutionnel des prix amont (prix du fromage à la fruitière) selon la valorisation finale du fromage $\left(\mathrm{NdC} \mathrm{n}^{\circ} 64, \mathrm{n}^{\circ} 71\right)$. L'interprofession CIGC joue un rôle essentiel dans le dispositif, en établissant et diffusant chaque mois un prix moyen pondéré des comtés vendus (la moyenne pondérée nationale comté $[\mathrm{MPnC}]$ ). Ce prix moyen pondéré permet d'établir le prix définitif des comtés en blanc achetés aux coopératives, en appliquant des coefficients négociés entre les fruitières et les affineurs (Jeanneaux et Perrier-Cornet, 2011, $\mathrm{NdC} \mathrm{n}^{\circ}$ 64, $\mathrm{n}^{\circ}$ 71). Pour que ce dispositif soit possible, la reconnaissance juridique a été essentielle ( $\left.\mathrm{NdC} \mathrm{n}^{\mathrm{o}} 18\right)$. En effet, pour que les plans de campagne soient aujourd'hui validés par le ministère des Finances (autorisation pour les pâtes pressées cuites), il a fallu longuement négocier, en lien (bridging) avec d'autres AOC dans le cadre de la Fédération nationale des appellations d'origine contrôlées (FNAOC) $\left(\mathrm{NdC}^{\circ} 31, \mathrm{n}^{\circ} 48\right)$. De façon plus structurelle, les décrets législatifs successifs de l'AOC comté sont un moyen de maîtriser l'offre à plus long terme, par exemple en fixant les limites de la zone de production (Jeanneaux et Perrier-Cornet, 2011). Cependant, enfin, le dispositif contractuel de régulation de l'offre ne permet pas en soi de fixer les volumes produits de façon coercitive. Pour qu'il puisse fonctionner, il est essentiel que les acteurs s'accordent pour « jouer le jeu », ce qui implique des normes sociales partagées autour de la coopération $\left(\mathrm{NdC} \mathrm{n}^{\mathrm{O}} 71\right)$. À l'échelle d'une fruitière, les liens forts entre agriculteurs (type bonding) permettent d'engager des niveaux élevés de concertation. Dans la perspective de la fin des quotas, par exemple, un outil innovant, « Modlait » $\left(\mathrm{NdC} \mathrm{n}^{\mathrm{o}} 83\right)$, a été récemment introduit pour gérer et anticiper collectivement les volumes de production à l'échelle des fruitières. Cet outil alterne des appuis techniques individuels avec des séances de synthèse collectives. Il permet par exemple de prendre conscience et d'anticiper les effets de la pyramide des âges d'une fruitière sur l'évolution des volumes produits $\left(\mathrm{NdC} \mathrm{n}^{\circ} 83\right)$.

\subsection{Mobilisation du capital social dans les domaines social et technique}

Dans le domaine social, le capital social a été mobilisé pour s'adapter aux évolutions majeures de la législation du travail depuis les années 1990 (travail le dimanche, passage aux 35 heures...). Ces évolutions remettaient profondément en cause l'organisation du travail du fromager, et par là même l'organisation de l'ensemble de la filière $\left(\mathrm{NdC}^{\circ}{ }^{\mathrm{O}} 57\right)$. En plus de la création de groupements d'employeurs entre plusieurs fruitières $\left(\mathrm{NdC} \mathrm{n}^{\circ} 57\right)$, la nature du capital social a permis d'offrir progressivement une diversité de solutions plus adaptées à chaque situation, comme l'embauche d'un second fromager, ou la vente ou l'échange du lait à une autre fromagerie $\left(\mathrm{NdC} \mathrm{n}^{\circ} 73\right.$, Tab. 1). Ces innovations se sont appuyées sur une connaissance et une reconnaissance du métier de fromager par les autres acteurs de la filière $\left(\mathrm{NdC} \mathrm{n}^{\circ} 7, \mathrm{n}^{\circ} 11\right.$, $\mathrm{n}^{\mathrm{o}} 54$ ), et la possibilité de poser le débat de façon franche (questions des compétences, des niveaux de salaires... $\mathrm{NdC}$ $\mathrm{n}^{\circ}$ 57). Dans le domaine technique, en plus des échanges continus entre les différents maillons de la filière et avec la recherche, certains mécanismes ont su tirer parti des liens forts entre les acteurs. Par exemple, pour inciter à la production de lait d'été (en vue d'obtenir des fromages à pâte jaune, plus riches en caroténoïdes), le CIGC a d'abord introduit un outil économique « vertical », la prime d'automne, d'un commun accord entre les représentants des coopératives laitières et des affineurs $\left(\mathrm{NdC} \mathrm{n}^{\circ} 6\right)$. Cette première tentative ayant été infructueuse (principalement pour des raisons d'organisation du travail des éleveurs), le CIGC a alors ouvert le débat plus « horizontal », de façon à ce que les producteurs discutent entre eux de la répercussion de la prime sur le prix du lait et organisent entre eux la complémentarité de leurs politiques de vêlage $\left(\mathrm{NdC} \mathrm{n}^{\mathrm{o}} 27\right)$. Autre exemple, pour favoriser l'autonomie des exploitations, des liens de type bridging sont entretenus avec les organismes de sélection de la race montbéliarde ( $\left.\mathrm{NdC} \mathrm{n}^{\circ} 86\right)$. Un prix « Meilleur élevage AOP », basé sur un indicateur d'autonomie, est attribué dans les comices $\left(\mathrm{NdC} \mathrm{n}^{\circ} 91\right)$. Les comices sont des lieux de socialisation privilégiés entre éleveurs et autres acteurs de la filière, où peuvent se forger et évoluer certaines valeurs sur ce qu'est « un bon élevage ».

\section{Discussion et conclusion}

Dans cette étude exploratoire, nous avons montré que le capital social de la filière comté est essentiel pour s'adapter à l'évolution de la demande par la maîtrise de l'offre, ainsi que pour d'autres problématiques dans les domaines sociaux et techniques. D'autres exemples auraient pu être apportés dans le domaine environnemental (notamment la valorisation de la biodiversité, $\mathrm{NdC} \mathrm{n}^{\circ} 19$ ). Pour bien comprendre cette contribution du capital social à une multiplicité de problématiques, la notion d'《 appropriabilité » des relations sociales nous paraît particulièrement éclairante. À l'origine des fruitières (dès le Moyen Âge), la collaboration entre producteurs était techniquement nécessaire pour valoriser le 
Tableau 1. Exemples d'activation des trois types de liens sociaux (bonding, linking, bridging) pour répondre à trois problématiques dans les domaines économiques, sociaux et techniques.

Table 1. Examples of activation of the three types of social links (bonding, linking, bridging) to deal with three issues in the economic, social and technical domains.

\begin{tabular}{|c|c|c|c|c|}
\hline $\begin{array}{l}\text { Nature du } \\
\text { lien }\end{array}$ & $\begin{array}{l}\text { Logique de proximité qui } \\
\text { prévaut / Nature et propriétés } \\
\text { des règles ratifiées (Angeon } \\
\text { et Callois, 2005) }\end{array}$ & $\begin{array}{l}\text { Maîtrise de l'offre pour } \\
\text { maintenir des prix élevés }\end{array}$ & $\begin{array}{l}\text { Évolution du travail des } \\
\text { fromagers }\end{array}$ & $\begin{array}{l}\text { Améliorations techniques } \\
\text { (qualité, autonomie) }\end{array}$ \\
\hline Bonding & $\begin{array}{l}\text { Logique de similitude / } \\
\text { Introjection de valeur }\end{array}$ & $\begin{array}{l}\text { Concertations entre éleveurs } \\
\text { (ex. : outil ModLait) } \\
\text { Volonté de « jouer le jeu» }\end{array}$ & & $\begin{array}{l}\text { Débat horizontal entre } \\
\text { producteurs (ex. : } \\
\text { complémentarité des } \\
\text { politiques de vêlage) }\end{array}$ \\
\hline Bridging & $\begin{array}{l}\text { Logique de relation à distance / } \\
\text { Convention sans engagement } \\
\text { réciproque }\end{array}$ & $\begin{array}{l}\text { Démarches pour la } \\
\text { reconnaissance juridique } \\
\text { (FNAOC) } \\
\text { Décrets de l'AOC comté } \\
\text { (limitations structurelles) }\end{array}$ & $\begin{array}{l}\text { (Re)connaissance du métier } \\
\text { de fromager par l'ensemble } \\
\text { des acteurs } \\
\text { Mise en débat à l'échelle } \\
\text { de la filière }\end{array}$ & $\begin{array}{l}\text { Échanges entre acteurs de } \\
\text { la filière } \\
\text { Collaborations (recherche } \\
\text { et développement, } \\
\text { sélection de la race } \\
\text { montbéliarde) } \\
\text { Prix du meilleur élevage } \\
\text { AOP (comices) }\end{array}$ \\
\hline
\end{tabular}

FNAOC : Fédération nationale des appellations d'origine contrôlées ; AOC : appellation d'origine contrôlée ; MPnC : moyenne pondérée nationale comté.

lait. À la période moderne, les liens sociaux hérités ont permis d'établir des mécanismes économiques sophistiqués, pour lesquels ils n'étaient pas prévus initialement. Ces mêmes liens sociaux, tout en se transformant, permettent aussi d'apporter des solutions innovantes dans les autres domaines.

Sur le plan méthodologique, le journal interne de l'interprofession nous a permis d'accéder à des éléments concrets pour les acteurs, souvent négligés par des analyses trop extérieures, comme la question du travail des fromagers. Cependant, pour aller plus loin, des indicateurs quantitatifs pourraient être imaginés, comme pour le capital social territorial (Angeon et Callois, 2005). Par ailleurs, la présente étude n'a pas abordé de fronts les aspects dynamiques du capital social. Pour la période récente, certains auteurs ont mis en garde contre des risques liés aux restructurations et aux phénomènes de concentration dans la filière (Kroll, 2008; Jeanneaux et al., 2011). Ces risques peuvent désormais être interprétés en termes d'érosion du capital social. Sans négliger ces risques d'érosion, il est important de souligner que le capital social permet de lutter, en quelque sorte, contre sa propre dégradation. En effet, la passion et la fierté sont extrêmement vives chez les différents acteurs à chaque niveau de la filière $\left(\mathrm{NdC} \mathrm{n}^{\circ} 44\right)$ et contribuent à expliquer le taux de renouvellement des générations particulièrement élevé $\left(\mathrm{NdC} \mathrm{n}^{\circ} 79\right)$. Il est aussi assez remarquable que les grands groupes extrarégionaux arrivés dans les années 1990 se soient globalement intégrés au modèle d'organisation sociale du comté (Fig. 1), dans une certaine mesure, en tissant des liens forts d'échange (et non de subordination) avec les coopératives. Des règles informelles $\left(\mathrm{NdC}^{\circ}{ }^{\circ} 63\right)$ et formelles (restriction du bassin de collecte des fruitières à un rayon de $25 \mathrm{~km}, \mathrm{NdC} \mathrm{n}^{\circ} 7$ ) ont contribué à cette insertion, en mobilisant pleinement le capital social. Selon les termes du président du CIGC Claude VermotDesroches, la filière a ainsi su évoluer sans perdre son « âme » $\left(\mathrm{NdC} \mathrm{n}^{\circ}\right.$ 69). Une approche chronologique serait intéressante pour étudier plus rigoureusement l'évolution des combinaisons de liens sociaux au cours du temps.

La filière comté possède sans doute un capital social particulièrement riche pour différentes raisons. Tout d'abord, la force de l'unité coopérative de base (la fruitière) bénéficie d'une remarquable profondeur historique. Dans ce sens, dans la lignée des travaux de Vernus (1988), Mélo (2015) définit la filière comté comme le produit d'une "société de voisins ", liée à une dynamique d'《 innovation perpétuelle » depuis les premières fruitières au XIII ${ }^{\mathrm{e}}$ siècle. Par ailleurs, l'interprofession CIGC a un rôle important dans la grande cohérence d'ensemble, en contribuant à maintenir vivaces les liens sociaux internes, et en sollicitant des réseaux extérieurs de façon opportune, comme pour la reconnaissance juridique. Pour Claude Vermot-Desroches, « La force [de la filière] c'est celle du village gaulois. Égoïsmes, orgueils mal placés et conflits à l'intérieur, mais organisation économique structurée autour du collectif et du respect mutuel, fondé sur un sentiment d'appartenance multiforme, à son village, à sa région, à sa fromagerie, à sa maison d'affinage, etc. » (Édito $\mathrm{NdC} \mathrm{n}^{\mathrm{o}} 79$ ).

Malgré le caractère spécifique de la filière comté prise comme illustration dans cette étude, nous pensons que l'importance du capital social vaut pour une diversité de situations et de filières laitières, y compris plus industrialisées. Pour ces autres filières, l'organisation de la filière comté peut sans doute donner matière à réflexion. Pourtant, cette filière comté ne saurait s'ériger en nouveau modèle à suivre ; au contraire, une leçon importante de sa réussite est que son développement vient « d'en bas », porté par les acteurs locaux. 
Dans une analyse de la coopération laitière dans l'ensemble de la montagne française, Ricard (2015) montre que si l'impulsion vient d'en haut (c'est-à-dire imposée de l'extérieur), les acteurs ne s'approprient pas le projet et celui-ci échoue. Autrement dit, le bon fonctionnement des relations sociales ne se planifie pas de l'extérieur : en montrant l'importance du capital social dans les capacités d'adaptation des filières laitières, nous défendons du même coup la pertinence d'un développement endogène porté par les acteurs locaux.

Remerciements. L'auteur tient à remercier deux relecteurs anonymes pour leurs remarques très pertinentes et détaillées qui ont permis d'enrichir considérablement ce travail. Merci également à Denise Renard du CIGC pour l'accès aux archives des Nouvelles du Comté.

\section{Références}

Adger WN. 2003. Social capital, collective action, and adaptation to climate change. Econ. Geogr. 79(4): 387-404.

Adler PS, Kwon SW. 2002. Social capital: prospects for a new concept. Acad. Manag. Rev. 27(1): 17-40.

Alguezaui S, Filieri R. 2010. Investigating the role of social capital in innovation: sparse versus dense network. J. Knowl. Manag. 14(6): 891-909.

Angeon V. 2008. L'explicitation du rôle des relations sociales dans les mécanismes de développement territorial. Rev. Econ. Reg. Urbaine 2: $237-250$.

Angeon V, Callois JM. 2005. Fondements théoriques du développement local : quels apports du capital social et de l'économie de proximité. Econ. Inst. 6-7: 19-49.

Angeon V, Caron P, Lardon S. 2006. Des liens sociaux à la construction d'un développement territorial durable : quel rôle de la proximité dans ce processus ? Développement durable et territoires (dossier 7), DOI: 10.4000/developpementdurable.2851

Chia E. 2008. La flexibilité relationnelle. Rôle des réseaux, groupements et associations d'éleveurs. In: Dedieu B, Chia E, Leclerc B, Moulin CH, Tichit M, eds. L'élevage en mouvement : flexibilité et adaptation des exploitations d'herbivores. Paris, France: Éditions Quae, pp. 135-142.

CICG. 2016. http://www.comte.com/le-kiosque/les-nouvelles-ducomte.html, dernière visite 18/07/2016.

Dedieu B, Ingrand S. 2010. Incertitude et adaptation : cadres théoriques et application à l'analyse de la dynamique des systèmes d'élevage. INRA Prod. Anim. 23(1): 81-90.

Dervillé M, Allaire G. 2014. Quelles perspectives pour les filières laitières de montagne après la suppression des quotas laitiers ? Une approche en termes de régime de concurrence. Spécificités de l'élevage de ruminants en montagne. In: Grosclaude J, Thibier M, Baumont R, eds. Dossier. INRA Prod. Anim. 27: 17-30.

Elisseef V. 2015. Le Comté : une réussite collective bâtie au fil des siècles. CIHEAM, Watch Letter $\mathrm{n}^{\mathrm{o}} 35$.

Fumey G, Bérion P. 2010. Dynamiques contemporaines d'un terroir et d'un territoire : le cas du gruyère de Comté. Ann. Geogr. 4: 384-403.

Jeanneaux P, Callois JM, Wouts C. 2009. Durabilité d'un compromis territorial dans un contexte de pression compétitive accrue. Le cas de la filière AOC Comté. Rev. Econ. Reg. Urbaine 1: 179-202.

Jeanneaux P, Perrier-Cornet P. 2011. Stratégie d'élévation des coûts des concurrents pour préserver un système productif agroalimentaire. Le cas d'une filière fromagère d'appellation d'origine. Rev. Econ. Ind. 135: 115-132.

Jeanneaux P, Meyer D, Barjolle D. 2011. Gouvernance des filières fromagères sous AOP et origine des prix de lait : un cadre d'analyse. Présenté lors du colloque. In: $5^{e}$ journées de recherches en Sciences sociales, Dijon (Vol. 8).

Kroll JC. 2008. La filière laitière de Franche-Comté. Rapport final d'étude ENESAD, 68 p.

Lin N. 2001. Building a theory of social capital. In: Cook KS, Lin N, Burt RS, eds. Social capital: theory and research. New Brunswick, New Jersey: Transaction Publishers.

Mélo A. 2015. « Fruitières comtoises ». De l'association de voisins au district agro-industriel : une histoire montagnarde ? J. Alp. Res. / Rev. Geogr. Alp.103-111, DOI: 10.4000/rga.2785

Putnam RD. 1995. Bowling alone: America's declining social capital. J. Democr. 6(1): 64-78.

Ricard D. 1994. Les montagnes fromagères françaises. ClermontFerrand: CERAMAC, deuxième édition, 1995, 496 p.

Ricard D. 2009. Qualité des produits et maîtrise des marchés : le cas des fromages et des AOC jurassiennes. Norois Environ., Aménage. Soc. 210: 91-105.

Ricard D. 2015. La coopération laitière dans la montagne française. Héritages, diversité des structures, dynamique du mouvement. J. Alp. Res. / Rev. Geogr. Alp.103-111, DOI: 10.4000/rga.2739

Rieutort L. 2006. Terrains de parcours, parcours de géographie : territoires d'élevage et espaces ruraux en France. HDR en géographie, Vol. 2. Clermont-Ferrand: Université Blaise-Pascal, 344 p.

Torre A. 2002. Les AOC sont-elles des clubs ? Réflexions sur les conditions de l'action collective localisée, entre coopération et règles formelles. Rev. Econ. Ind. 100(1): 39-62.

Vernus M. 1988. Le comté, une saveur venue des siècles. Paris: Édition Textel.

Woolcok M, Narayan D. 2000. Social capitals: implication for development theory, research and policy. World Bank Res. Obs. 15 (2): 225-249.

Citation de l'article: Rigolot C. 2016. Le capital social des filières : une dimension essentielle de leurs capacités d'adaptation. Illustration avec la filière comté. Cah. Agric. 25: 45007. 